\title{
THE INFLUENCE OF MEASUREMENT ERRORS ON THE COSMOLOGICAL DECELERATION PARAMETER OBTAINED FROM THE SEMIRELATIVISTIC MODEL OF COLLECTIVE GALACTIC MOTION
}

\author{
S. L. Parnovsky ${ }^{1}$, A. S. Parnowski ${ }^{2}$ \\ ${ }^{1}$ Astronomical Observatory of Kyiv Taras Shevchenko National University, \\ Observatorna St., 3a, Kyiv, 04053,Ukraine, e-mail: par@observ.univ.kiev.ua \\ ${ }^{2}$ Space Research Institute NASU $\&$ NSAU, \\ prosp. Akad. Glushkova, 40, block 4/1, Kyiv-187, 03680 MSP, Ukraine \\ e-mail:parnowski@gmail.com \\ (Received March 15, 2010)
}

\begin{abstract}
We use Monte Carlo simulations to demonstrate that the deviations of the parameters of the semirelativistic model of collective motion of flat edge-on spiral galaxies are caused by measurement errors in angular diameters and HI line widths of galaxies. This effect is related to the well-known Malmquist bias.
\end{abstract}

Key words: large-scale galaxy motion, statistical methods, cosmology.

PACS number(s): 98.62.Py; 98.80.Es; 02.70.Uu

\section{INTRODUCTION}

At the present time the Universe is essentially inhomogeneous on the scales of about 10-100 Mpc. The development of initial fluctuations led to an observable large-scale structure. The regions with increased matter density provide an additional attraction of surrounding galaxies. The regions with decreased density, e.g. voids, also make an input to the collective large-scale motion of galaxies on the background of Hubble expansion. The investigation of such motion allows on the one hand to map the matter density, including dark matter, in the Local Universe, and on the other hand its parameters are linked with cosmological parameters.

In 1989 Karachentsev [1] proposed to use flat edgeon spiral galaxies as a tool for studying their large-scale collective motion. They are good in this role for the following reasons:

1. The linear diameter is strongly correlated with the $\mathrm{HI}$ linewidth for thin bulgeless galaxies. This allows to determine distances without photometric data.

2. Flat galaxies can be easily identified by their axial ratio.

3. Flat galaxies have a nearly $100 \% \mathrm{HI}$ detection rate.

4. Galaxies in clusters are usually not flat due to the interaction with neighbours. This means that flat galaxies avoid clustering and do not interact with the intergalactic gas in clusters.

5. Peculiar velocities of isolated flat galaxies are not perturbed by neighbours.

To implement this idea the Flat Galaxy Catalogue (FGC, [2]) was created. It contained data about 4455 galaxies, which satisfied the conditions $a_{b} / b_{b} \geq 7$ and $a_{b}>0.6^{\prime}$. Here $a_{b}$ and $b_{b}$ are the major and minor axial diameters directly measured on POSS-I and ESO/SERC plates. In accordance with the original photographic material, the Catalogue consists of two parts: FGC (2573 galaxies) and its southern extension, FGCE (1882 galaxies). The first part is based on the POSS-I and covers the sky region with declinations between $-20 \mathrm{deg}$ and $+90 \mathrm{deg}$. The second one is based on the ESO/SERC and covers the rest of the sky area up to $\delta=-90 \mathrm{deg}$.

After thorough studies of the catalogue's properties, both these parts were joined. The angular diameters from the FGCE were converted to the POSS-I system of the FGC, which appeared to be close to the $a_{25}$ system. This system where galaxy size is taken at $B=25 \mathrm{mag} / \square^{\prime \prime}$ isophotal level was used, in particular, by deVacouleurs et al. [3]. Some FGCE galaxies, which did not satisfy the condition $a>0.6^{\prime}$ after conversion, were removed from the resulting Revised Flat Galaxy Catalogue (RFGC, [4]). It contained data about 4236 galaxies including the information on the following parameters: Right Ascension and Declination for the epochs J2000.0 and B1950,0, galactic longitude and latitude, major and minor blue and red diameters in arcminutes in the POSS-I diameter system, morphological type of the spiral galaxies according to the Hubble classification, index of the mean surface brightness (I - high, IV - very low) and some other parameters, which are not used in this article. A more detailed description of the catalogue can be found in [4].

The original goal of this catalogue was to estimate the distance to galaxies according to the Tully-Fisher relationship in the "HI line width - linear diameter" version without using their redshifts. The difference between the velocity $V$ derived from the redshift and the Hubble velocity $R=H r$ corresponding to the distance $r$ estimated by Tully-Fisher relationship is called a peculiar velocity $V_{\mathrm{pec}}=c z-H r$.

There are some important things to take into account about peculiar velocities. The redshift includes not on- 
ly the velocity of the galaxy, but also the velocities of our Galaxy, the Solar System and the Earth. Thus, to eliminate these factors, all velocities were reduced to the frame, where CMB is isotropic. Naturally, the redshift gives us only the radial component of the velocity and the tangential components cannot be measured. Additionally, Tully-Fisher relationship is statistical and thus has a certain error. Hence, we can only estimate the peculiar velocity for each galaxy, sometimes with a significant error.

However, we believe that there is a large-scale velocity field. We consider the individual galaxies as test particles in the velocity field of a large-scale collective motion. Thus, having data about the peculiar velocities of a large number of galaxies, we can restore the radial component of the velocity field. For this reason we need ample samples of peculiar velocities, preferably uniformly covering the celestial sphere.

These conditions are satisfied by the RFGC catalogue, which covers the whole celestial sphere in both hemispheres with the natural exclusion of the Milky Way region. However, not all of the RFGC galaxies have data required to estimate the distance to them using the TullyFisher relationship. Nevertheless, the sample of galaxies having such data is also quite uniform.

To apply the Tully-Fisher relationship the data from the catalogue is not sufficient; we also need to know the HI linewidth $W$ (in this article we take it at $50 \%$ level), or the gas rotation velocity $V_{\text {rot }}$ obtained from optical observations. Additionally, we need redshift data. The number of galaxies with such data increases constantly.

Since the time when FGC and RFGC were assembled a number of articles have been published dealing with collective motions of RFGC galaxies. Very preliminary results were published by Karachentsev et al. [5]. The parameters of the bulk motion were calculated by Karachentsev et al. [6]. In [7] not only new data were added, but also the models featuring the quadrupole and octopole components of the velocity field were proposed. Also, the generalized Tully-Fisher relationship for RFGC galaxies was finalized. It included data not only about HI linewidth and angular diameter in red and blue bands, but also about the morphological type of the galaxy and its surface brightness index. By that time the authors had information about radial velocities and $\mathrm{HI} 21 \mathrm{~cm}$ line widths or $V_{\text {rot }}$ for 1327 RFGC galaxies from different sources listed in [6]. From this number, 1271 galaxies were included in the sample, and the rest of them were considered to be outliers. As a result, the first list of peculiar velocities of RFGC galaxies was prepared by Karachentsev et al. [8]. Four years later, the number of galaxies with available data increased and reached 1561 [9]; 1493 of them entered the sample. In this article we will use this sample.

Analyzing the velocity field of the collective motion by multipole decomposition we constructed the described below model of galaxy motion. This model was constructed for flat space. However, according to GTR, our spacetime is curved and even in the case of homogeneous isotropic Universe the actual galaxy velocity de- viates from the simple Hubble law. Considering the next term of the decomposition in $R$ we obtain

$$
V=R+\gamma R^{2}
$$

For distances $R$ measured by angular diameters the value $\gamma$ can be expressed through the cosmological deceleration parameter $q$ in the following way, see e.g. [10]:

$$
\gamma=\frac{3+q}{2 c}=3.98 \times 10^{-6} \mathrm{skm}^{-1} .
$$

We used the value of $q$ from the standard $\Lambda$ CDM model

$$
q=\frac{\Omega_{m}}{2}-\Omega_{\Lambda}=-0.61,
$$

where $\Omega_{m}$ and $\Omega_{\Lambda}$ are relative densities of matter including dark matter and dark energy, respectively. Numerical estimations are based on the results of 7-year WMAP observations [11]. Note that for different methods of determination of distances $R$, e.g. photometric distances, the expression for $\gamma$ will be also different.

In the case when curved space-time contains an inhomogeneous matter distribution, cosmic flows appear which must be described in the GTR framework. The corresponding research was performed by Kudrya and Alexandrov $[12,13]$. It was applied to real data in paper [14]. The results have shown that the value of $\gamma$ calculated from the peculiar velocities data of RFGC galaxies essentially differed from estimation (1). The obtained value was $(-18.4 \pm 2.4) \times 10^{-6} s \mathrm{~km}^{-1}$. Note that its sign is also opposite to that in equation (1). In this article we show a possible reason behind this effect. This is a purely statistical effect related to Malmquist bias. It is caused by errors in measurement of angular diameters and HI line widths. We also use Monte Carlo simulations to demonstrate that the value obtained by Parnovsky and Gaydamaka [14] can be explained both qualitatively and quantitatively.

\section{MULTIPOLE MODELS OF COLLECTIVE MOTION OF GALAXIES}

In [7] the velocity field components in the CMB reference frame were expanded in terms of galaxy's radial vector $\mathbf{r}$. The first three terms are

$$
V_{i}=D_{i}+A_{i k} r_{k}+O_{i k l}^{\prime} r_{k} r_{l}
$$

In our notation we use the Einstein rule: summation by all the repeating indices. Hence, for the radial velocity $V_{3 K}$ in the CMB reference frame we get

$$
V_{3 K}=D_{i} n_{i}+r A_{i k} n_{i} n_{k}+r^{2} O_{i k l}^{\prime} n_{i} n_{k} n_{l},
$$

where $\mathbf{n}=\mathbf{r} / r$ and $r=|\mathbf{r}|$. Let us decompose the tensor $\mathbf{A}$ into two parts: $A_{i k}=H \delta_{i k}+Q_{i k}^{\prime}$. Here $H$ is a trace, corresponding to the Hubble constant, $\delta_{i k}$ is the Kronecker delta, and $\mathbf{Q}^{\prime}$ is a traceless tensor. Next we switch from distance $r$ to the corresponding Hubble velocity $R=H r$.

$$
V_{3 K}=R+D_{i} n_{i}+R Q_{i k} n_{i} n_{k}+R^{2} O_{i k l} n_{i} n_{k} n_{l},
$$


where $\mathbf{Q}=H^{-1} \mathbf{Q}^{\prime}$ and $\mathbf{O}=H^{-2} \mathbf{O}^{\prime}$.

This decomposition was used to obtain some models of dependence of galaxy's radial velocity $V$. In the simplest D-model (Hubble law + dipole) we have

$$
V_{3 K}=R+V^{\mathrm{dip}}+\delta V, V^{\mathrm{dip}}=D_{i} n_{i},
$$

where $\mathbf{D}$ is a velocity of homogeneous bulk motion, $\delta V$ is a random deviation and $\mathbf{n}$ is a unit vector towards a galaxy. After the addition of quadrupole terms we obtain a DQ-model

$$
V_{3 K}=R+V^{\mathrm{dip}}+V^{\text {qua }}+\delta V, V^{\text {qua }}=R Q_{i k} n_{i} n_{k}
$$

with the symmetrical traceless tensor $\mathbf{Q}$ describing quadrupole components of the velocity field. It characterises the relative deviation of an effective "Hubble constant" in a given direction from the mean value. The DQOmodel includes the octopole component of velocity field described by the symmetrical tensor $\mathbf{O}$ of rank 3:

$$
\begin{aligned}
& V_{3 K}=R+V^{\text {dip }}+V^{\text {qua }}+V^{\text {oct }}+\delta V, \\
& V^{\text {oct }}=R^{2} O_{i k l} n_{i} n_{k} n_{l} .
\end{aligned}
$$

In some cases it makes sense to use another way of describing the octopole component in the DQO-model. For this purpose the tensor $\mathbf{O}$ can be considered a sum of a traceless tensor $\hat{\mathbf{O}}$, and a trace, characterized by the vector $\mathbf{P}$

$$
O_{i j k}=\hat{O}_{i j k}+P_{(i} \delta_{j k)}, P_{i}=\frac{3}{5} O_{i j k} \delta_{j k} .
$$

Here the indices in parantheses are symmetrized. Thus an alternative form of the DQO-model is

$$
\begin{aligned}
V_{3 K}= & R+\left(D_{i}+R^{2} P_{i}\right) n_{i}+R Q_{i k} n_{i} n_{k} \\
& +R^{2} \hat{O}_{i k l} n_{i} n_{k} n_{l}+\delta V .
\end{aligned}
$$

We will use Cartesian components of the vector $\mathbf{n}$ in the galactic coordinates:

$$
\begin{aligned}
& n_{1}=n_{z}=\sin b, n_{2}=n_{x}=\cos l \cos b, \\
& n_{3}=n_{y}=\sin l \cos b .
\end{aligned}
$$

These three models were used for processing data on RFGC galaxies. To estimate the distances to galaxies a generalized Tully-Fisher relationship was used in the 'linear diameter - HI line width' version. It has the form [7]

$$
\begin{aligned}
R= & \left(C_{1}+C_{2} B+C_{3} B T+C_{4} U\right) \frac{W}{a} \\
& +C_{5}\left(\frac{W}{a}\right)^{2}+C_{6} \frac{1}{a},
\end{aligned}
$$

where $W$ is a corrected HI line width in $\mathrm{km} \mathrm{s}^{-1}$ measured at $50 \%$ of the maximum, $a$ is a corrected major galaxies' angular diameter in arcminutes on red POSS and $\mathrm{ESO} / \mathrm{SERC}$ reproductions, $U$ is a ratio of major galaxies' angular diameters on red and blue reproductions, $T$ is a morphological type indicator $\left(T=I_{t}-5.35\right.$, where $I_{t}$ is a Hubble type; $I_{t}=5$ corresponds to type $\mathrm{Sc}$ ), and $B$ is a surface brightness indicator $\left(B=I_{S B}-2\right.$, where $I_{S B}$ is a surface brightness index from RFGC; brightness decreases from I to IV). Details of corrections one can find in [7,9]. The reasons for choosing this form of Tully-Fisher relationship for RFGC galaxies are given ibid. We only note that the statistical significance of each term in eq. (12) is greater than $99 \%$.

The D-model has 9 parameters $(6$ coefficients $C$ and 3 components of vector D), DQ-model has 14 parameters (5 components of tensor $\mathbf{Q}$ are added), and DQOmodel is described by 24 coefficients. The values and errors of the coefficients were calculated by the least square method for different subsamples with distances limitation to make the sample more homogeneous in depth [7]. We used the same weights for all datapoints. Since the quadrupole and octopole terms explicitly depend on $R$, an iteration procedure was used. Note that the coefficients of the generalised Tully-Fisher relationship and components of the velocity model were fitted simultaneously. The iterations converge rather quickly.

The situation in the curved space is more complex. It as was shown by Kudrya and Alexandrov $[12,13]$ the expression for the velocity should be modified in the following way:

$$
V_{3 K}=R+V^{\text {dip }}+V^{\text {qua }}+V^{\text {oct }}+\gamma R^{2} .
$$

Since we moved the terms proportional to $R^{2}$ into a separate term we should remove them from the Tully-Fisher relation:

$$
R=\left(C_{1}+C_{2} B+C_{3} B T+C_{4} U\right) \frac{W}{a}+C_{5} \frac{1}{a},
$$

We also should introduce an additional term to the octopole component of the velocity: $V^{\text {oct }}=$ $R^{2}\left(P_{i} n_{i}+O_{i j k} n_{i} n_{j} n_{k}+S_{i j} n_{i} n_{j}\right)$, where $S_{i j} n_{i} n_{j}=$ $s_{1}\left(n_{1}^{2}-n_{3}^{2}\right)+s_{2}\left(n_{2}^{2}-n_{3}^{2}\right)+s_{3} n_{1} n_{2}+s_{4} n_{1} n_{3}+s_{5} n_{2} n_{3}$ is a symmetric traceless tensor. The dipole and quadrupole components remain the same as in the nonrelativistic case.

In $[12,13]$ it was shown that the relation

$$
\begin{aligned}
C_{\alpha \beta \gamma \delta} V^{\beta} V^{\delta} & =\frac{H^{2}}{c^{2}}\left[-2 c S_{\alpha \gamma}+6 Q_{\alpha \gamma}\right. \\
& \left.-3 Q_{\alpha}^{\epsilon} Q_{\epsilon \gamma}-\left(V_{\alpha} V_{\gamma}-g_{\alpha \gamma} Q_{\epsilon \eta} Q^{\epsilon \eta}\right)\right]
\end{aligned}
$$

must hold. Here Greek indices denote four-dimensional components, $g_{\alpha \beta}$ is a metrical tensor, and $C_{\alpha \beta \gamma \delta}$ is a Weyl tensor. The spatial parts of four-dimensional tensors $Q_{\alpha \beta}$ and $S_{\alpha \beta}$ coincide with three-dimensional tensors $\mathbf{Q}$ and $\mathbf{S}$, and their temporal and mixed parts are much smaller. The four-dimensional velocity vector can be assumed equal to $\left(g_{00}^{-1 / 2}, 0,0,0\right)$. For the homogeneous isotropic Universe Weyl tensor and tensors $\mathbf{S}$ and $\mathbf{Q}$ vanish. They are connected with spatial inhomogeneities of the density distribution, e.g. attractors and voids. The left part of this relation is a sum of inputs of individual inhomogeneities. For a spherically symmetric attractor with an excessive mass $M$ at a distance $L$ the spatial part of the tensor $C_{\alpha \beta \gamma \delta} V^{\beta} V^{\delta}$ after reduction to eigenaxes receives the form:

$$
C_{\alpha \beta \gamma \delta} V^{\beta} V^{\delta}=\xi\left(\begin{array}{ccc}
2 & 0 & 0 \\
0 & -1 & 0 \\
0 & 0 & -1
\end{array}\right),
$$


where $\xi=G M / c^{2} L^{3}$.

In [14] the value of this tensor was estimated using approximate excessive masses and distances to the nearest attractors. The result was that the $\mathbf{S}$ value obtained with the least squares method exceeds the expected value by several orders. This is connected with the fact that the terms containing the tensors $\mathbf{Q}$ and $\mathbf{S}$ have the same distribution over the celestial sphere. Due to measurement errors and deviations from the Tully-Fisher relation the tensor S "borrows" some of the value of tensor $\mathbf{Q}$. Unfortunately, with the quality and quantity of observational data at hand we are unable to correctly separate the inputs of these tensors. Thus, taking into account the small values of tensor $\mathbf{S}$, the so-called semirelativistic model was introduced in [14], which differs from the full relativistic model only by the dropped term with tensor $\mathbf{S}$. However, in both these models the obtained values of $\gamma$ appear to be way off the expected value, namely: $-16.1 \pm 2.7$ in the relativistic model and $-18.4 \pm 2.4$ in the semirelativistic model. This inconsistence will be addressed in the next chapters.

\section{ESTIMATING THE IMPACT OF MEASUREMENT ERRORS: A SIMPLE CASE}

Before trying to address this problem at its full extent, let us consider a simple case where an analytical solution can be provided. Let us start off from introducing a toy model $y=A x+B x^{2}$, where $x=W / a$ is a main term of the Tully-Fisher relation (14), $y=V, A=C_{1}$, and $B=\gamma_{0} C_{1}^{2}$, where $\gamma_{0}$ is a true value of $\gamma$. This model corresponds to an isotropic Hubble expansion with cosmological acceleration.

Observational data provide us with a set of $N$ points characterized by values $x_{i}$ and $y_{i}$. It is important to realize how measurement errors and deviations from the Tully-Fisher relation distort the dataset. The errors in velocity measurements and deviations from Tully-Fisher relation yield errors in $y$. The values of $A$ and $B$ determined by the least square method from the dataset with such errors will have normal distribution of errors without shift. At the same time, errors in measurements of $W$ or $a$ yield errors in $x$. This case is often called Malmquist bias in statistics. It is usually applied to a linear dependence, but the general effects will be the same. Due to this effect, the perceived values of $A$ and $B$ given by the least square method will have a systematic error.

Let us consider the following case: we have $N$ values of $x_{i}$ distributed uniformly over the interval $[0,1]$ with a step $(N-1)^{-1}$. The values of $y$ are calculated with $A=A_{0}=1$ and $B=B_{0}$. In each of $N$ points the $x_{i}$ is shifted by $\sigma \xi_{i}$, where $\xi_{i}$ is a normally distributed quantity with zero mean and unit variance. The different values of $\xi_{i}$ are not correlated with each other. The values $y_{i}$ are calculated from the original nondisplaced values of $x_{i}$. The values of $A$ and $B$ given by the least square method have the form

$$
\begin{gathered}
A=\frac{\sum_{i=1}^{N} y_{i} x_{i} \sum_{i=1}^{N} x_{i}^{4}-\sum_{i=1}^{N} y_{i} x_{i}^{2} \sum_{i=1}^{N} x_{i}^{3}}{\sum_{i=1}^{N} x_{i}^{2} \sum_{i=1}^{N} x_{i}^{4}-\left(\sum_{i=1}^{N} x_{i}^{3}\right)^{2}}, \\
B=\frac{\sum_{i=1}^{N} x_{i}^{2} y_{i} \sum_{i=1}^{N} x_{i}^{2}-\sum_{i=1}^{N} x_{i}^{3} \sum_{i=1}^{N} x_{i} y_{i}}{\sum_{i=1}^{N} x_{i}^{2} \sum_{i=1}^{N} x_{i}^{4}-\left(\sum_{i=1}^{N} x_{i}^{3}\right)^{2}}, \\
x_{i}^{0}=\frac{i-1}{N-1}, \quad x_{i}=x_{i}^{0}+\sigma \xi_{i}, \quad y_{i}=x_{i}^{0}+\gamma_{0}\left(x_{i}^{0}\right)^{2} .
\end{gathered}
$$

It is not very difficult to calculate the mean values of $A$ and $B$ over $\xi$ using the following expressions:

$$
\begin{aligned}
& A=A_{1}+\gamma_{0} A_{2}, \\
& B=B_{1}+\gamma_{0} B_{2} .
\end{aligned}
$$

Here we designated

$$
\begin{aligned}
& A_{1}=\frac{1+40 \sigma^{2}+60 \sigma^{4}}{1+28 \sigma^{2}+180 \sigma^{4}+720 \sigma^{6}}, \\
& A_{2}=\frac{28 \sigma^{2}+60 \sigma^{4}}{1+28 \sigma^{2}+180 \sigma^{4}+720 \sigma^{6}}, \\
& B_{1}=\frac{-20 \sigma^{2}+120 \sigma^{4}}{1+28 \sigma^{2}+180 \sigma^{4}+720 \sigma^{6}}, \\
& B_{2}=\frac{1-43 / 3 \sigma^{2}+80 \sigma^{4}}{1+28 \sigma^{2}+180 \sigma^{4}+720 \sigma^{6}} .
\end{aligned}
$$

Note that the latter formulae are precise up to $\mathcal{O}(1 / N)$. To verify these formulae we calculated $A$ and $B$ for this toy model using 10000 Monte Carlo simulations. The mean values perfectly fitted the given formulae (see Figure 1).

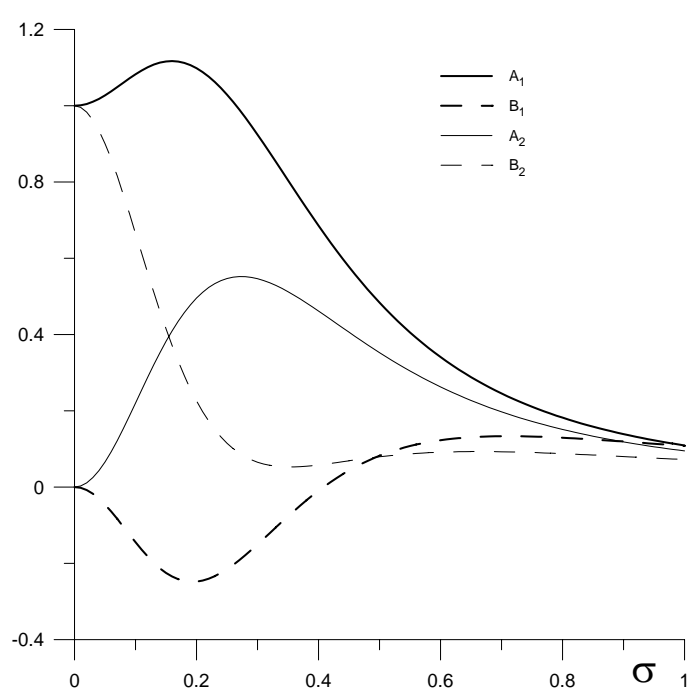

Fig. 1. Dependence of coefficients of equations (19) and (20) on the noise level. 


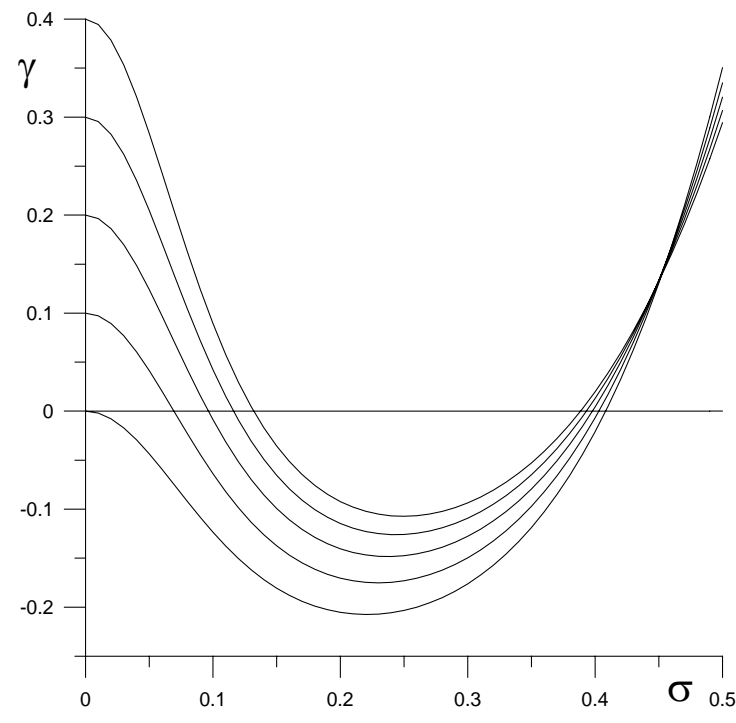

Fig. 2. Dependence of least squares estimation of $\gamma$ on the noise level for different values of $\gamma_{0}$. The values of $\gamma_{0}$ equal to values of $\gamma$ at $\sigma=0$.

If we calculate $\gamma$ using the formula $\gamma=B / A^{2}$, we will obtain instead of the true value $\gamma_{0}$ a value $\gamma$, plotted on Figure 2 against $\sigma$ for different $\gamma_{0}$. At $\sigma=0$, i.e. when there are no errors, we obtain $\gamma=\gamma_{0}$, but at small $\sigma$ we obtain $\gamma<\gamma_{0}$. This is the impact of the measurement errors we demonstrate.

\section{ESTIMATING THE IMPACT OF MEASUREMENT ERRORS: A REAL CASE}

The actual case is much more complicated. The problem cannot be reduced to the one-dimensional case since the galaxy's velocity depends on its position on the celestial sphere. To estimate the distance we use all the terms in relation (14). The errors in the determination of distances are non-Gaussian. They are due to errors in angular diameters and HI line widths and deviations from the Tully-Fisher relation. These errors were analyzed in paper [15]. Here we will briefly mention the main results of this analysis. These errors can be described by four parameters:

$$
\begin{gathered}
V_{i}=V_{i}^{(0)}\left(1+s_{V} \xi_{1}\right), \\
W_{i}=W_{i}^{(0)}\left(1+s_{W} \xi_{2}\right), \\
a_{i}=a_{i}^{(0)}\left(1+s_{a} \xi_{3}\right)+\Delta_{a} \xi_{4} .
\end{gathered}
$$

Here $\xi_{1}, \xi_{2}, \xi_{3}$, and $\xi_{4}$ are the four independent noncorrelating random values. They are distributed according to Gauss law with zero mean and unit variance.

Let us describe what errors correspond to each type of noise. The noise (25) describes the deviations from the Tully-Fisher relationship. It does not include velocity measurement error because radial velocities are well determined, as well as the directions towards galaxies. Such a type of noise provides a conventional log-normal distribution of the velocity deviation. If this noise is used alone, the maximal value of $s_{V}$ can be assumed about 0.2 , which corresponds to $20 \%$ uncertainty of distance estimated by the Tully-Fisher relation.

The noise (26) corresponds to HI line width measurement errors. Different methods of calculating HI halfwidth of the same profile can differ by up to $10 \%$. Thus, if this noise is used alone, the maximal value of $s_{W}$ can be set to 0.15 with some tolerance.

The noise (27) describes angular diameter measurement errors. The value $s_{a}$ describes a relative error caused by variations of exposition, curvature of galaxies, etc. The diameter measurements have an accuracy of not more than 5-10\% (Karachentseva, private communication). The value $\Delta_{a}$ describes an absolute error of measurement. This value is important for the smallest galaxies. Since these diameters were measured in tenths of millimetre, which corresponded to 0.11 arcmin, the maximal value of $\Delta_{a}$ can be estimated between 0.05 and 0.1 . Note that if we use non-zero values of $s_{a}$ and $\Delta_{a}$ simultaneously, the maximum estimation of $\Delta_{a}$ should be somewhat reduced to avoid overestimating the noise added to angular diameters for the smallest galaxies.

Naturally, such a difficult problem of determining the shift of $\gamma$ cannot be solved analytically. We use Monte Carlo simulations to resolve it numerically. Let us describe the details of this procedure.

We use some sample of galaxies. In our case this is a subsample of $N=1357$ galaxies, whose distance in the nonrelativistic D-model is less than $100 h^{-1} \mathrm{Mpc}$. For each of the galaxies we substitute the measured radial velocity with the radial velocity calculated using the formulae $(13,14,6,7,8)$. We use the values given by equations (1) and (2).

After that we add noise to our model and for each of 10000 simulations we calculate the coefficients in the same way as we treat actual data, i. e. using the semirelativistic DQO-model. Thus, for each realisation we obtain a complete set of the coefficients including $\gamma$. For the obtained values of $\gamma$ we calculate the mean and the standard deviation. Taking into account that the distribution of $\gamma$ is non-Gaussian, its quantiles differ from the ones calculated from the normal distribution. In this article we give errors corresponding to the $99 \%$ confidence level. Application of Monte Carlo method allows to do this in a straightforward way. From 10000 values of $\gamma$ for different realizations we find the 50th largest and smallest values. They give us the boundaries of the $99 \%$ confidence interval.

In principle, we can apply this procedure to any mock catalogue. However, we should use a catalogue that has the same spatial distribution as well as a distribution of morphological types, surface brightness index, etc. as the main sample. The best mock catalogue is thus the sample itself. We use as a result the sample of actually measured parameters, namely angular diameter, surface brightness index, Hubble type, HI line width, ratio of angular diameters in red and blue imprints, and celestial coordinates. 
The radial velocity is calculated from formulae $(13,14)$ with the coefficients obtained from the real sample. Theoretically, we could use distances to galaxies calculated with the coefficients of the semirelativistic model by Parnovsky and Gaydamaka [14], but we use a more refined procedure.

At first we calculated the coefficients for the semirelativistic model using real data while fixing the value of $\gamma$ at $\gamma_{0}$. Then we used this set of coefficients to calculate "radial velocities" for the galaxies. Such "radial velocities" are closer to the actual radial velocities than for any other models with fixed $\gamma$.

The values obtained using the Monte Carlo method for different parameters of the noise are given in Table 1 . The top part of Table 1 illustrates the impact of each individual type of noise. One can see that the $\gamma$ value acts in the same way as in the simple case considered in the previous section. The noise $s_{V}$, corresponding to the deviations from the statistical Tully-Fisher relation has little or no effect on the value of $\gamma$. All other types of noise lead to a drastic reduction of $\gamma$, especially the noise $\Delta_{a}$. The bottom part of Table 1 contains the results obtained with realistic noise parameters. When choosing parameters we used as a control parameter the $\sigma$ value for the noised sample, comparing it to the value $\sigma=1125 \mathrm{~km} \mathrm{~s}^{-1}$ for real data. This parameter is convenient because it vanishes in the absence of the noise and grows when the noise increases. Thus, it can help avoiding undernoising and overnoising. Other constraints used and details of the procedure are described in [15]. Naturally, we do not try to find a unique set of noise parameters. The four noise values form a four-dimensional parameter space, the $\sigma$ constraint yields a three-dimensional hypersurface in it. Other constraints give us rough estimates of the boundaries of the volume of suitable values.

One can see from Table 1 that for a set of parameters lying inside or close to those boundaries, the value of $\gamma$ is much less than the initial value $\gamma_{0}$. Comparing these values with the value $\gamma=(-18.4 \pm 2.4) \times 10^{-6} \mathrm{~s} \mathrm{~km}^{-1}$ obtained by Parnovsky and Gaydamaka [14] from the real data, we can select a range of suitable noise parameters. For many realistic noise parameters the value of $\gamma$ falls into the $1 \sigma$ area, and for most of them it falls into $99 \%$ confidence area. In those cases when $\gamma$ misses the $99 \%$ confidence area, the value of $\Delta_{a}$ is unrealistically large.

\begin{tabular}{|c|c|c|c|c|r|r|r|}
\hline & & & & & \multicolumn{3}{|c|}{$\gamma, 10^{-6} \mathrm{sm}^{-1}$} \\
\cline { 6 - 8 }$s_{V}$ & $s_{W}$ & $s_{a}$ & $\Delta_{a}$ & $\sigma, \mathrm{km} \mathrm{s}^{-1}$ & $\langle\gamma\rangle \pm \sigma_{\gamma}$ & \multicolumn{2}{|c|}{$99 \% \mathrm{CL}$} \\
\cline { 6 - 8 } & & & & & & & \\
\hline 0.05 & 0.00 & 0.00 & 0.00 & $282 \pm 7$ & $4.0 \pm 1.2$ & 1.0 & 7.3 \\
0.10 & 0.00 & 0.00 & 0.00 & $564 \pm 14$ & $4.1 \pm 2.5$ & -1.9 & 10.8 \\
0.15 & 0.00 & 0.00 & 0.00 & $845 \pm 22$ & $4.2 \pm 3.7$ & -4.5 & 14.4 \\
0.20 & 0.00 & 0.00 & 0.00 & $1127 \pm 29$ & $4.4 \pm 5.1$ & -7.2 & 19.3 \\
0.00 & 0.05 & 0.00 & 0.00 & $306 \pm 8$ & $-0.1 \pm 1.2$ & -2.8 & 3.1 \\
0.00 & 0.10 & 0.00 & 0.00 & $584 \pm 14$ & $-8.1 \pm 1.5$ & -11.7 & -3.9 \\
0.00 & 0.15 & 0.00 & 0.00 & $821 \pm 20$ & $-14.9 \pm 1.4$ & -18.0 & -11.0 \\
0.00 & 0.20 & 0.00 & 0.00 & $1021 \pm 24$ & $-19.3 \pm 1.2$ & -22.0 & -15.9 \\
0.00 & 0.00 & 0.05 & 0.00 & $278 \pm 7$ & $-1.7 \pm 1.1$ & -4.4 & 1.0 \\
0.00 & 0.00 & 0.10 & 0.00 & $534 \pm 13$ & $-13.2 \pm 1.2$ & -16.2 & -9.7 \\
0.00 & 0.00 & 0.15 & 0.00 & $753 \pm 18$ & $-22.1 \pm 0.9$ & -24.3 & -19.6 \\
0.00 & 0.00 & 0.20 & 0.00 & $947 \pm 24$ & $-26.2 \pm 1.0$ & -28.1 & -22.3 \\
0.00 & 0.00 & 0.00 & 0.05 & $368 \pm 12$ & $-7.4 \pm 1.4$ & -11.0 & -3.6 \\
0.00 & 0.00 & 0.00 & 0.10 & $662 \pm 20$ & $-20.6 \pm 1.3$ & -23.4 & -15.3 \\
\hline 0.10 & 0.15 & 0.05 & 0.05 & $1063 \pm 25$ & $-20.5 \pm 1.4$ & -23.8 & -16.6 \\
0.10 & 0.20 & 0.05 & 0.05 & $1217 \pm 28$ & $-23.1 \pm 1.2$ & -26.0 & -19.6 \\
0.10 & 0.15 & 0.07 & 0.07 & $1116 \pm 26$ & $-23.4 \pm 1.1$ & -26.0 & -20.2 \\
0.12 & 0.15 & 0.05 & 0.05 & $1127 \pm 27$ & $-20.5 \pm 1.5$ & -24.1 & -16.2 \\
0.15 & 0.12 & 0.05 & 0.05 & $1155 \pm 28$ & $-18.3 \pm 2.0$ & -22.9 & -12.7 \\
0.13 & 0.15 & 0.05 & 0.03 & $1138 \pm 27$ & $-18.4 \pm 1.8$ & -22.7 & -13.1 \\
0.17 & 0.10 & 0.05 & 0.03 & $1166 \pm 28$ & $-13.5 \pm 2.6$ & -19.6 & -5.7 \\
\hline
\end{tabular}

Table 1. Results of Monte Carlo simulations. 


\section{CONCLUSION}

We demonstrated that the deviation of the parameter $\gamma$ from the parameter $\gamma_{0}=3.98 \times 10^{-6} \mathrm{~s} \mathrm{~km}^{-1}$ following from the cosmological parameters can be explained both qualitatively and quantitatively by measurement errors in angular diameters and HI line widths of galaxies. From the statistical point of view, this effect is related to the Malmquist bias. However, since this particular case is too complex to be treated analytically we used Monte Carlo to demonstrate this.

Additionally, according to equation (13), the shift of $\gamma$ will lead to the overestimation of radial distances to the galaxies by $18 \%$ for the noise with $s_{V}=0.13, s_{W}=0.15$, $s_{a}=0.05$ and $\Delta_{a}=0.03$.

\section{ACKNOWLEDGEMENTS}

The authors are grateful to L. S. Pilyugin for valuable criticism.
[1] I. D. Karachentsev, Astron. J. 97, 1566 (1989).

[2] I. D. Karachentsev et al., Astron. Nachr. 314, 97 (1993).

[3] G. de Vaucouleurs et al., Third Reference Catalogue of Bright Galaxies (Berlin-Heidelberg-New York, Springer, 1991).

[4] I. D. Karachentsev et al., Bull. SAO 47, 5 (1999) (astro$\mathrm{ph} / 0305566)$.

[5] I. D. Karachentsev et al., Astron. Nachr. 316, 369 (1995).

[6] I. D. Karachentsev et al., Astron. Rep. 44, 150 (2000).

[7] S. L. Parnovsky et al., Astron. Lett. 27, 765 (2001).

[8] I. D. Karachentsev et al., Bull. SAO 50, 5 (2000) (astroph/0107058).

[9] S. L. Parnovsky, A. V. Tugay, Astron. Lett. 30, 357
(2004).

[10] Ia. B. Zeldovich, I. D. Novikov, Relativistic astrophysics (Chicago, University of Chicago Press, 1983).

[11] E. Komatsu et al., arXiv:1001.4538 (2010).

[12] Yu. N. Kudrya, A. N. Alexandrov, J. Phys. Stud. 6, 472 (2002).

[13] Yu. N. Kudrya, A. N. Alexandrov, Herald Kyiv Univ. Astron. 39-40, 130 (2004). (in Ukrainian)

[14] S. L. Parnovsky, O. Z. Gaydamaka, Kinem. Phys. Celest. Bodies 20, 477 (2004).

[15] S. L. Parnovsky, A. S. Parnowski, Astron. Nachr. 329, 864 (2008).

\title{
ВПЛИВ ПОХИБОК ВИМІРЮВАННЯ НА ПАРАМЕТР КОСМОЛОГІЧНОГО УПОВІЛЬНЕННЯ, ОТРИМАНОГО В НАПІВРЕЛЯТИВІСТСЬКІЙ МОДЕЛІ КОЛЕКТИВНИХ РУХІВ ГАЛАКТИК
}

\author{
С. Л. Парновський ${ }^{1}$, А. С. Парновський ${ }^{2}$ \\ ${ }^{1}$ Астрономічна обсерваторія Київсъкого національного університету імені Тараса Шевченка, \\ вул. Обсерваторна, 3а, Київ, 04053, Україна \\ ${ }^{2}$ Інститут космічних досліджень НАНУ $і$ НКАУ, \\ nросп. акад. Глушкова, 40, корп. 4/1, Київ-187, 03680, Україна
}

За допомогою методу Монте-Карло ми демонструємо, що відхилення параметрів напіврелятивістської моделі колективних рухів пласких спіральних галактик, видимих із ребра, викликані похибками вимірювання кутових діаметрів та ширин лінії НІ галактик. Цей ефект споріднений із добре відомим статистичним ефектом Малмквіста. 